\title{
DISTRIBUCIÓN, ABUNDANCIA Y DIETA DE Pteronura brasiliensis LOBO DE Río EN LA CUENCA DE LOS RÍOS PENEYA Y ANGUSILLA, PARQUE NACIONAL GÜEPPÍ SEMIKE
}

\author{
Frank FLORES PONCE ${ }^{1}$, Fatima SANCHEZ MOSQUEDA ${ }^{2}$, Johana DEZA GRADOS ${ }^{3}$ \\ 1 Wildlife Conservation Society WCS. Urb. Sargento Lores Mz “0” Lote 1. Iquitos, Perú. fflores@wcs.org \\ 2 Universidad Nacional de la Amazonia Peruana. Av. Elias Aguirre № 1342. Iquitos, Perú. fatimasanchez_21@hotmail.com \\ 3 World Wildlife Fund WWF. Calle Trinidad Morán N853. Lima, Perú. johana.deza@wwfperu.org
}

\section{RESUMEN}

Con el objetivo de contribuir a la conservación del lobo de río Pteronura brasiliensis en el Parque Nacional Güeppí Semike, evaluamos la distribución, abundancia y dieta de esta especie en los ríos Peneya y Angusilla entre los meses de julio a octubre del 2012. Su distribución y abundancia fueron determinadas a través de observaciones de evidencias directas e indirectas en recorridos de transectos acuáticos, en tanto que su dieta a través de la colecta y analisis de restos de heces. Se reportó un total de 11 avistamientos, 42 individuos (26 en la cuenca del río Peneya y 16 en el río Angusilla) y 65 observaciones indirectas entre madrigueras, campamentos, letrinas, heces y huellas. La abundancia relativa del lobo de río en la cuenca del río Peneya fue de $0,29 \mathrm{ind} . / \mathrm{km}$, mientras que en la cuenca del río Angusilla fue de $0,23 \mathrm{ind} . / \mathrm{km}$; además, se estima un individuo por cada $3,45 \mathrm{~km}$ y 4,29 km de recorrido para la cuenca de los ríos Peneya y Angusilla, respectivamente. La dieta de Pteronura brasiliensis fue $100 \%$ de peces, representado por 4 órdenes (Characiformes, Perciformes, Siluriformes y Cupleiformes) para la cuenca Peneya, y 2 órdenes (Characiformes y Perciformes) para la cuenca del río Angusilla. Satanoperca jarupari, perteneciente al orden Perciformes, familia Cichlidae, fue la especie más frecuentemente registrada en el análisis de dieta.

PALABRAS CLAVE: Pteronura brasiliensis, Ecología, Conservación, Alimentación, Población.

\section{DISTRIBUTION, ABUNDANCE AND DIET OF Pteronura brasiliensis GIANT OTTER IN THE PENEYA AND ANGUSILLA RIVERS, GÜEPPÍ SEMIKE NATIONAL PARK}

\begin{abstract}
In order to contribute to the conservation of the giant otter Pteronura brasiliensis in Güeppí Semike National Park, we evaluated the distribution, abundance and diet of this species in the Peneya and Angusilla rivers between July and October of 2012. Its distribution and abundance were determined through observations of direct and indirect evidences in aquatic transect routes, while its diet through the collection and analysis of feces. A total of 11 sightings, 42 individuals (26 in the Peneya River and 16 in the Angusilla River) and 65 indirect observations among burrows, camps, latrines, feces and footprints were reported. The relative abundance of the giant otter was $0.29 \mathrm{ind} . / \mathrm{km}$ in the Peneya river basin, while it was $0.23 \mathrm{ind} . / \mathrm{km}$ in the Angusilla river basin. In addition, an individual is estimated for each $3.45 \mathrm{~km}$ and $4.29 \mathrm{~km}$ of route for these rivers, respectively. The diet of Pteronura brasiliensis was $100 \%$ fish, corresponding to 4 orders (Characiformes, Perciformes, Siluriformes and Cupleiformes) in the Peneya river basin, and 2 orders (Characiformes and Perciformes) in the Angusilla river basin. Satanopercajarupari, of the order Perciformes and family Cichlidae, was the species most frequently recorded in the diet analysis.
\end{abstract}

KEYWORDS: Pteronura brasiliensis, ecology, conservation, feeding, population. 


\section{INTRODUCCIÓN}

Pteronura brasiliensis (Zimmerman, 1780) lobo de río, es un mamífero semiacuático social y diurno endémico de Sudamérica. Se alimenta principalmente de peces y está adaptado morfológicamente para nadar (Carter \& Rosas, 1997; Rosas et al., 1999; Cabral et al., 2010).

Es una especie de importancia para los ecosistemas acuáticos, siendo considerada bioindicadora de la integridad acuática, es gregaria, carismática y principalmente diurna. Se encuentra en el tope de la cadena alimenticia y las acciones para su conservación también benefician aquellas especies ubicadas en los estratos inferiores (Isola \& Benavides, 2001).

Entre las décadas del cuarenta y sesenta del siglo pasado, esta especie fue perseguida indiscriminadamente debido al valor comercial de su piel poniendo en peligro de extinción a la especie (Staib \& Schenck, 1994). Actualmente, el lobo de río está incluida en el Apéndice I del CITES, y en el Perú está considerada una especie en peligro de extinción (EN), y protegida por el Estado peruano según Decreto Supremo No 004-2014-MINAGRI.

En el 2011, en la cuenca de los ríos Peneya y Angusilla, se identificó un potencial conflicto entre pescadores de las comunidades y el lobo de río (WWF, 2011), debido a que según los pescadores, los lobos de río son consideradas como competidor por los peces, en especial con Osteoglossum bicirrhosum arahuana, recurso pesquero de valor comercial para las comunidades (Flores, 2012).

El presente trabajo tuvo como objetivo conocer la distribución, abundancia y dieta de lobo de río durante la época de vaciante intermedia en las cuencas de los ríos Peneya y Angusilla, con miras a tomar acciones para la conservación de esta especie en la zona.

\section{MATERIAL Y MÉTODOS}

\section{ÁREA DE ESTUDIO}

El trabajo fue realizado en el Parque Nacional de Güeppí Semike, situada al extremo norte del Perú, cercana a la línea ecuatorial y limitada por los ríos Putumayo y Lagartococha. La zona es uno de los lugares con mayor diversidad en el mundo (Alverson et al., 2008).

Fueron seleccionadas las cuencas de los ríos Peneya y Angusilla, afluentes del río Putumayo (margen derecha), aguas arriba y abajo respectivamente de la localidad de Soplín Vargas, Distrito Teniente Manuel Clavero, Provincia de Maynas, Región Loreto.

\section{DISTRIBUCIÓN Y ABUNDANCIA DEL LOBO DE RÍO}

El trabajo se llevó a cabo entre agosto y octubre del 2012. Para esto, se realizaron recorridos en bote o canoa por "cochas" principales y quebradas. Algunas veces fue necesario realizar caminatas alrededor de las cochas cuando el acceso era imposible debido al bajo nivel del río y presencia de árboles caídos. Por los cuerpos de agua de la cuenca del río Peneya se realizaron recorridos de $89,6 \mathrm{~km}$ y por la cuenca del río Angustilla se realizaron recorridos de $68,7 \mathrm{~km}$. Se determinó la ocurrencia espacial de la especie, en términos de presencia o ausencia (Groenendijk et al., 2005), utilizando la técnica de transectos acuáticos barriendo cada tramo. Se registró, georeferenció y fotografió toda evidencia directa e indirecta. Las evidencias directas fueron constituidas por avistamiento de la especie y las indirectas por visualizaciones de madriguera, campamento, letrina, huellas u otro. Los recorridos en la cuenca de los ríos Peneya y Angusilla se realizaron en bote con motor $15 \mathrm{HP}$ a velocidad mínima y en canoa desde las 5:30 a.m. hasta las 5:00 p.m. Se registró observaciones directas del lobo de río utilizando la técnica de barrido, y éstas fueron georeferenciadas con un GPS MAP 60s.

\section{DIETA DE Pteronura brasiliensis}

Fueron colectadas heces de las madrigueras y campamentos. Para ello se utilizaron bolsas de polietileno herméticas, las mismas que fueron debidamente rotuladas. Las heces colectadas fueron conservadas en formol al $10 \%$ y posteriormente enviadas al Laboratorio de Taxonomía de Peces del Instituto de Investigaciones de la Amazonía Peruana (IIAP), donde se realizaron el análisis e identificación de restos sólidos. Para esto, las muestras fueron separadas en grupos de escamas según su morfología (ctenoidea, cicloidea, tamaño, forma). Las escamas colectadas fueron comparadas con muestras conservadas del laboratorio. Se extrajo de 3-5 escamas por especie o género, siempre teniendo en cuenta que se encuentren en el rango del tamaño de alimentación de la especie (entre 10 y 35 $\mathrm{cm}$ ); además se colectó en campo las escamas de una arahuana encontrada muerta en la zona de estudio. Con las escamas extraídas del laboratorio se realizó una lista de especies con su respectiva muestra al costado, las cuales sirvieron para comparar con las muestras de escamas de las heces de lobo de río y de esa manera determinar la familia y en algunos casos hasta género de los peces encontrados en las heces.

\section{PROCESAMIENTO Y ANÁLISIS DE DATOS}

Los datos obtenidos tanto para el uso de hábitat, 
alimentación y dieta, densidad y abundancia del lobo de río y los conocimientos y formas de percibir de la población fueron ordenados y procesados en las hojas de cálculo del programa EXCEL. El análisis de los datos se realizaron utilizando estadísticas descriptivas con la ayuda del programa estadístico BIOESTAT. La estimación de la abundancia relativa se calculó utilizando la siguiente fórmula: $A=N / L$, donde: $\mathrm{A}=$ Abundancia relativa, $\mathrm{N}=$ Número total de individuos registrados, $\mathrm{L}=$ Distancia total recorrida.

\section{RESULTADOS}

\section{DISTRIBUCIÓN Y ABUNDANCIA DEL LOBO DE RÍO}

En la cuenca del río Peneya se registraron un total de 7 avistamientos y 26 individuos observados, (Figura 1, Tabla 1) lo cual se complementó con observaciones indirectas entre madrigueras, campamentos, letrinas y huellas (Tabla 2). En la cuenca del río Angusilla se registraron 04 avistamientos y 16 individuos observados (Tabla 2). Las evidencias indirectas estaban bien relacionadas con las observaciones y no en todos los recorridos hubo avistamientos. Se encontró un total de 6 madrigueras, 2 campamentos, 3 letrinas y 7 huellas; pero la mayoría de ellas se distribuían hasta el sector denominado Sabalillo, después de ello casi no se encontró evidencia de lobos de río hasta la repartición del río Angusilla con el río Huiririma (Figura 2, Tabla 1).

Para el río Peneya se estima una abundancia relativa de 0,29 ind./ $/ \mathrm{km}$ y se calcula que existe un individuo por cada $3,45 \mathrm{~km}$; mientras que en río Angusilla se estima que la abundancia relativa es de 0,23 ind. $/ \mathrm{km}$ y se calcula la existencia de un individuo por cada $4,29 \mathrm{~km}$ de recorrido.

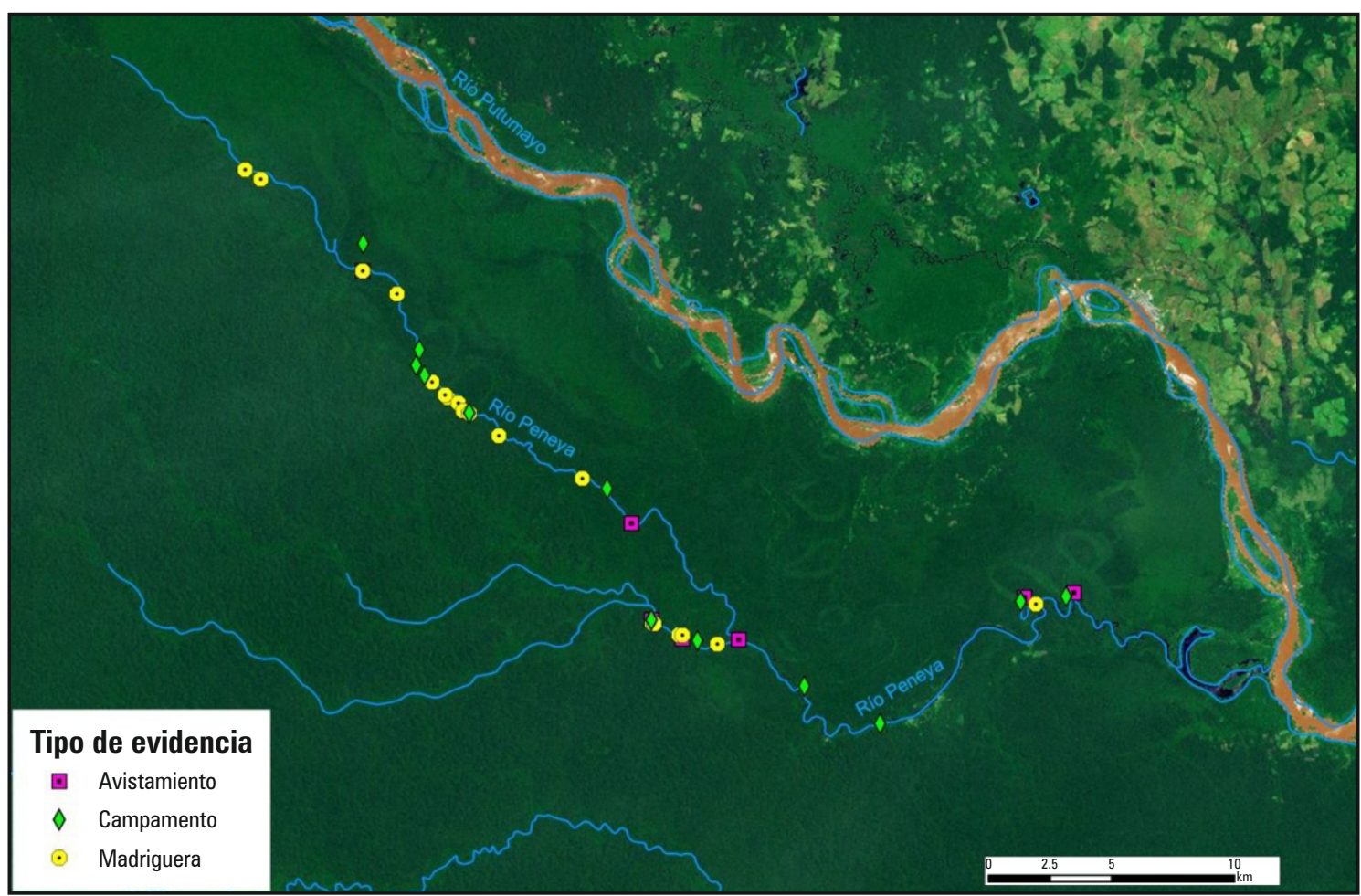

Figura 1. Distribución de Pteronura brasiliensis en la cuenca del río Peneya (Parque Nacional Güeppí Semike). 
Tabla 1. Avistamientos del lobo de río Pteronura brasiliensis en la cuenca Peneya y Angusilla (Parque Nacional Güeppí Semike).

\begin{tabular}{ccccc}
\hline Cuenca & Avistamiento & Lugar & Sector & $\begin{array}{c}\text { Numero de } \\
\text { individuos }\end{array}$ \\
\hline & 1 & Río Peneya & Repartición & 4 \\
Río & 2 & Quebrada aguas negras & Salao & 2 \\
Peneya & 3 & Quebrada aguas negras & Primera Cocha & 5 \\
& 4 & Río Peneya & Boa Caño & 5 \\
& 5 & Río Peneya & Caño Negro & 8 \\
\hline Río & 6 & Quebrada aguas blancas & - & 1 \\
\hline Angusilla & 7 & Quebrada aguas blancas & - & 1 \\
\hline & 1 & Río Angusilla & Cocha Huitoto & 5 \\
\hline & 3 & Río Angusilla & Isango & 2 \\
\hline
\end{tabular}

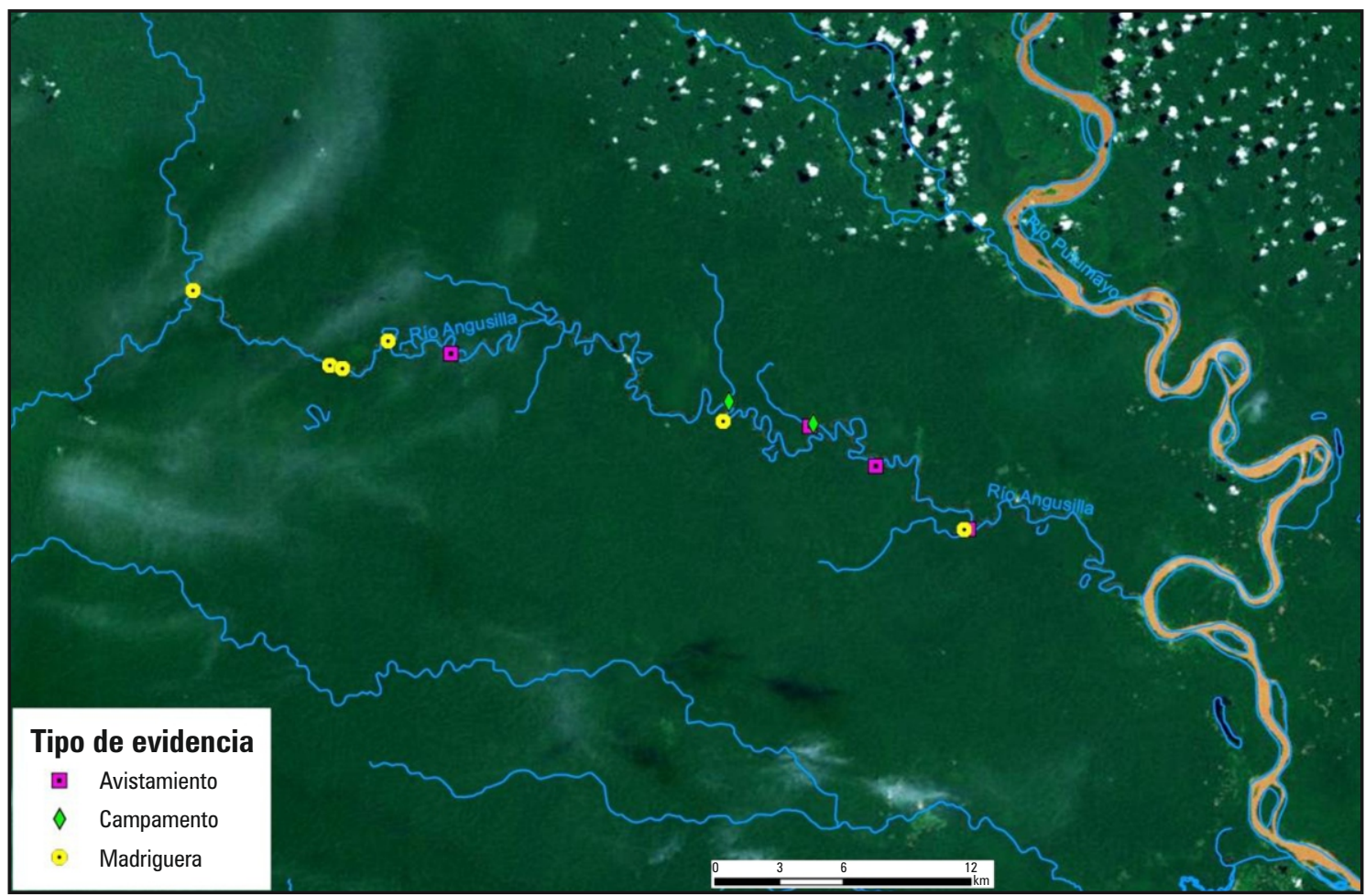

Figura 1. Distribución de Pteronura brasiliensis en la cuenca del río Angusilla (Parque Nacional Güeppí Semike). Fuente: Elaborado por WWF (2012) con los datos colectados por el autor. 


\section{DIETA DEL LOBO DERÍO}

El 100\% de la dieta estuvo compuesta por peces (tablas 3 y 4), donde destacan los órdenes Characiformes, Clupeiformes, Perciformes y Siluriformes en la cuenca Peneya y sólo Characiformes y Perciformes en la cuenca Angusilla.

Tabla 3. Base de datos mostrando las especies de peces identificados en las muestras de heces del lobo de río en la cuenca Peneya.

\begin{tabular}{|c|c|c|}
\hline CóDIGO & ORDEN & FAMILIA \\
\hline HE001 & CHARACIFORMES & Prochilodontidae \\
\hline HE001 & CHARACIFORMES & Prochilodontidae \\
\hline HE001 & PERCIFORMES & Cichlidae \\
\hline HE001 & PERCIFORMES & Cichlidae \\
\hline HE002 & CHARACIFORMES & Characidae \\
\hline HE002 & CHARACIFORMES & Characidae \\
\hline HE002 & CHARACIFORMES & Erythrinidae \\
\hline HE003 & CHARACIFORMES & Characidae \\
\hline HE003 & CHARACIFORMES & Characidae \\
\hline HE003 & CHARACIFORMES & Characidae \\
\hline HE003 & CHARACIFORMES & Erythrinidae \\
\hline HE003 & CLUPEIFORMES & Pristigasteridae \\
\hline HEOO3 & PERCIFORMES & Cichlidae \\
\hline HE004 & CHARACIFORMES & Anostomidae \\
\hline HE005 & CHARACIFORMES & Anostomidae \\
\hline HE006 & CHARACIFORMES & Characidae \\
\hline HE006 & SILURIFORMES & Doradidae \\
\hline HE007 & CHARACIFORMES & Characidae \\
\hline HE008 & CHARACIFORMES & Anostomidae \\
\hline HE008 & PERCIFORMES & Cichlidae \\
\hline HE008 & PERCIFORMES & Cichlidae \\
\hline HE009 & CHARACIFORMES & Anostomidae \\
\hline HEOO9 & PERCIFORMES & Cichlidae \\
\hline HE010 & PERCIFORMES & Cichlidae \\
\hline HE011 & PERCIFORMES & Cichlidae \\
\hline HE012 & CHARACIFORMES & Acestrorhynchidae \\
\hline HE012 & PERCIFORMES & Cichlidae \\
\hline HE013 & CHARACIFORMES & Erythrinidae \\
\hline HE013 & CHARACIFORMES & Prochilodontidae \\
\hline HE013 & PERCIFORMES & Cichlidae \\
\hline HE014 & CHARACIFORMES & Characidae \\
\hline HE014 & PERCIFORMES & Cichlidae \\
\hline
\end{tabular}

Tabla 4. Base de datos mostrando las especies de peces identificados en las muestras de heces del lobo de río en la cuenca Angusilla.

\begin{tabular}{lll}
\hline CÓDIGO & \multicolumn{1}{c}{ ORDEN } & \multicolumn{1}{c}{ FAMILIA } \\
\hline HE001 & CHARACIFORMES & Characidae \\
HE001 & CHARACIFORMES & Characidae \\
HE001 & CHARACIFORMES & Anostomidae \\
HE001 & CHARACIFORMES & Erythrinidae \\
HE001 & PERCIFORMES & Cichlidae \\
HE001 & PERCIFORMES & Cichlidae \\
HE002 & CHARACIFORMES & Anostomidae \\
HE002 & CHARACIFORMES & Prochilodontidae \\
HE003 & CHARACIFORMES & Characidae \\
HE003 & CHARACIFORMES & Prochilodontidae \\
\hline
\end{tabular}

De las 17 muestras encontradas, 14 pertenecen a la cuenca del río Peneya; y de las especies de peces encontradas, el orden Characiformes fue el más frecuente con un $65 \%$, seguido por el orden Perciformes con $23 \%$, finalmente los órdenes Siluriformes y Clupeiformes con $6 \%$ cada uno. Además, el orden Characiformes presentó la mayor cantidad de familias con 5: Acestrorhynchidae, Anostomidae, Characidae, Erythrinidae y Prochilodontidae; mientras que los otros 3 órdenes presentaron sólo una familia cada uno (Cichlidae, Pristigasteridae y Doradidae respectivamente) siendo Cichlidae la familia con mayor frecuencia. De las 03 muestras, los peces encontrados en la cuenca Angusilla, la mayoría perteneció al orden Characiformes con un $78 \%$ y presentó además la mayor cantidad de familias presentes (Anostomidae, Characidae, Erythrinidae y Prochilodontonidae), mientras que el orden Perciformes estuvo presente en un $22 \%$ sólo con la familia Cichlidae.

\section{DISCUSIÓN}

Durante el trabajo de investigación se reportó la presencia de $P$. brasiliensis en la cuenca de los ríos Peneya y Angusilla; y consolida lo reportado por Carter y Rosas (1997) y Duplaix (1980) quienes mencionan su distribución en esta zona de América del sur por debajo de $600 \mathrm{msnm}$. Se registró un total de 42 individuos y 65 observaciones indirectas entre madrigueras, campamentos, letrinas y huellas en ambas cuencas y concuerda con los resultados reportados por Alverson et al. (2008) y Nuñez (2010) quienes también reportan esta especie para estos ríos. Además, se tuvo una distribución 
uniforme en toda el área trabajada y también muy cercana a las comunidades de Santa Teresita y Santa Rosa de Escalante, lo cual sugiere que las poblaciones de $P$. brasiliensis se encuentran en buen estado; mientras que en el río Angusilla las observaciones sean directas o indirectas se encuentran más aisladas, es decir, son menos uniformes, esto posiblemente debido a la mayor presencia de pobladores por los alrededores y al menor esfuerzo en cuanto a réplicas durante el trabajo de campo.

En término de abundancia, se estima que existe un individuo por cada $3,45 \mathrm{~km}$ y $4,29 \mathrm{~km}$ de recorrido para la cuenca de los ríos Peneya y Angusilla respectivamente, indicando poblaciones muy saludables en ambas cuencas, con un ligero mejor estado en la cuenca del río Peneya. Además, estos resultados presentan mejor estado en las poblaciones del lobo de río que los reportados por Schenck (1999) para el Parque Nacional Manú, que reporta un individuo por cada $5,7 \mathrm{~km}$ de recorrido y aún más por los reportados por Recharte (2006) en el río Yavarí y Samiria con 6,29 ind./km y 5,82 ind./km; lo cual indica que las poblaciones del lobo de río se encuentran ligeramente más abundantes en la Zona Reservada de Güeppí que en las dos anteriores zonas mencionadas.

En las muestras de heces encontradas, el 100\% corresponde a peces pertenecientes a cuatro órdenes (Characiformes, Perciformes, Siluriformes y Clupeiformes) en la cuenca del río Peneya y muestra relación con lo reportado por Carrasquilla (2002), Damasceno (2004), Recharte (2006) y Hoffmann (2007) quienes mencionan estos 4 órdenes en la dieta del lobo de río. En complemento, Duplaix (1980), Gómez-Serrano (1999), Laidler (1984), Velasco (2004) reportan también la presencia de peces en más del $90 \%$, pero agregan en la dieta grupo de crustáceos, anfibios, reptiles y mamíferos pequeños. En la cuenca del río Angusilla sólo se reportó la presencia de peces de los órdenes Characiformes y Perciformes, posiblemente debido a la menor cantidad de muestras de heces colectadas en esta cuenca.

En relación a los órdenes, Characiformes estuvo mayormente presente en las muestra con $65 \%$ para el río Peneya, seguido por Perciformes y en menor porcentaje Siluriformes y Clupeiformes; lo cual concuerda con lo observado por Duplaix (1980), Carrasquilla (2002), Damasceno (2004), Velasco (2004) Recharte (2006) y Hoffmann (2007) quienes mencionan un alto porcentaje del orden Characiformes. Se reportaron 8 familias de peces (Acestrorhynchidae, Anostomidae, Characidae, Cichlidae, Doradidae, Erythrinidae,
Pristigasteridae, Prochilodontonidae) al igual que la mayoría de los autores que tienen reportado en mayor cantidad y casi siempre las familias Anostomidae, Characidae, Cichlidae, Erythrinidae. La familia Cichlidae fue la más predominante en las muestras con $64,3 \%$ y coincide con lo reportado por Damasceno (2004) quien menciona a Cichlidae como la más frecuente, con Velasco (2004) quien reporta a Cichlidae con un $67,6 \%$ de sus muestras como el más frecuente, y Recharte (2006) quien menciona que Cichlidae fue la familia más abundante con $41 \%$.

\section{AGRADECIMIENTOS}

Nuestro agradecimiento especial a World Wildlife Fund Inc. WWF-Perú por el apoyo técnicofinanciero y a Hewlett Packard por el financiamiento del proyecto. Igualmente al equipo de trabajo de la Zona Reservada de Güeppí, categorizada a Parque Nacional Güeppí-Sekime y Reservas Comunales Airo Pai y Huimeki en octubre del 2012; en especial al Jefe del Parque Nacional Güeppí-Sekime, el Sr. Teófilo Torres, y los guardaparques que contribuyeron en las salidas de campo: David Yumbo, Juan Pacaya, Uriel Talexio, Elmer Panaifo, Joel Miranda y Humberto Ibáñez. Especial gratitud para los pobladores de las comunidades nativas Santa Teresita, Santa Rosa de Escalante y Mashunta, y a sus respectivos caciques Mauro Caimito, Belisario Llomero y Neiser Garcés. De igual manera agradecer el apoyo del Blgo. Homero Sánchez, responsable del laboratorio de Taxonomía de peces e investigador del IIAP.

\section{BIBLIOGRAFÍA CITADA}

Alverson, W.; Vriesendorp, C.; Del Campo, Á.; Moskovits D.; Stotz D.; García M.; Borbor L. 2008. Ecuador-Perú: Cuyabeno-Güeppí. Rapid Biological and Social Inventories Report. The Field Museum, Chicago. 376 pp.

Carrasquilla, M. C. 2002. Uso de hábitat, comportamiento y dieta de la nutria gigante (Pteronura brasiliensis) en río Orinoco, Vichada, Colombia. Tesis de pre-grado. Universidad de Los Andes, Facultad de Ciencias, Bogotá. 62 pp.

Carter, S.K.; Rosas, F.C. 1997. Biology and Conservation of the Giant Otter Pteronura brasiliensis. Mammal Review, 27(1): 1-26.

Damasceno, J. 2004. Estudos Ecológicos de ariranha, Pteronura brasiliensis, (Zimmermann, 1780) (Carnivora: Mustelidae) no Pantanal Mato Grossense. Universidade Federal de Mato 
Grosso-Cuiabá. Instituto de Biociências, Cuiabá. $76 \mathrm{pp}$.

Duplaix, N. 1980. Observations on the ecology and behaviour of the giant otter Pteronura brasiliensis in Suriname. Revue Ecologique Terre Vie, 34(1): 495-620.

Flores, H. 2012. Diagnóstico de la situación actual del lobo de río en la cuenca Peneya - Zona Reservada de Güeppí. Santa Teresita-Perú. World Wildlife Fund, Lima. 39 pp.

Gómez-Serrano, J. R. 1999. Ecología alimentaria de la nutria gigante (Pteronura brasiliensis) en el bajo Río Bita Vichada, Colombia. Tesis de pregrado, Pontificia Universidad Javeriana, Facultad de Ciencias, Bogotá. 50 pp.

Groenendijk, J.; Hajek, F.; Duplaix, N.; Reuther C.; Van Damme, P.; Schenck C.; Staib E.; Wallace R.; Waldemarin H.; Notin R.; Marmontel M.; Rosas F.; Mattos G.; Evangelista E.; Utreras V.; Lasso G.; Jacques H.; Matos K.; Roopsind I.; Botello J. 2005. Surveying and monitoring distribution and population trends of the giant otter (Pteronura brasiliensis). Guidelines for a standardisation of survey methods as recommended by the giant otter section of the IUCN/SSC Otter Specialist Network. Habitat, 16:1-100.

Hoffmann, I. 2007. The behavioural and ecological requirements of a recovering giant otter (Pteronura brasiliensis) population: Buen Fin oxbow lake in the Lago Preto Concessionary Reserve, Loreto, Peru 2006-2007. Tesis de pregrado, University of Kent, Canterbury, Reino Unido. $78 \mathrm{pp}$.

Isola, S. Benavides, J. 2001. El Lobo de río: Una especie bandera para la reserva comunal Tamshiyacu Tahuayo. Wildlife Conservation Society, Lima. 45 pp.
Laidler, P.E. 1984. The behavioural ecology the giant river otter in Guyana. Tesis de doctorado, University of Cambridge, Cambridge, Reino Unido. 296 pp.

Nuñez, R. 2010. Evaluación preliminar del "lobo de río" Pteronura brasiliensis (Gmelin, 1788) en las cuencas Peneya y Angusilla de la Zona Reservada de Güeppí, Loreto, Perú. SERNANP, Lima. 19 pp.

Recharte, M. 2006. Evaluación Poblacional del lobo de río (Pteronura brasiliensis, Zimmermann, 1780) en la cuenca de los ríos Yavarí y Samiria, Loreto-Perú. Tesis de pre-grado, Universidad Nacional de la Amazonia Peruana, Facultad de Ciencias Biológicas, Iquitos. 135 pp.

Staib, E.; Schenck, C. 1994. Status, habitat use and conservation of giant otter in Peru, In: Dunstone, N.; Gorman, M. L. (Eds.) Behavior and Ecology of Riparian Mammals: 71th Simposio of Zoological Society of London. p. 1-20.

Schenck, C. 1999. Lobo de río: presencia, uso de hábitat y protección en el Perú. GTZ, INRENA, Lima. 174 pp.

Velasco, D. 2004. Valoración biológica y cultural de la nutria gigante (Pteronura brasiliensis), en el área de influencia de Puerto Carreño, Vichada, Colombia (Ríos Orinoco, Bita, Caños Juriepe y Negro). Tesis de pregrado. Pontificia Universidad Javeriana, Facultad de Estudios Ambientales y Rurales, Bogotá. 89 pp.

WWF. 2011. Manejo pesquero y el lobo de río. (www.wwfperu.org). Acceso 02/04/2012.

Recibido: 10 de Junio del 2016

Aceptado para publicación: 17 de Julio del 2016 


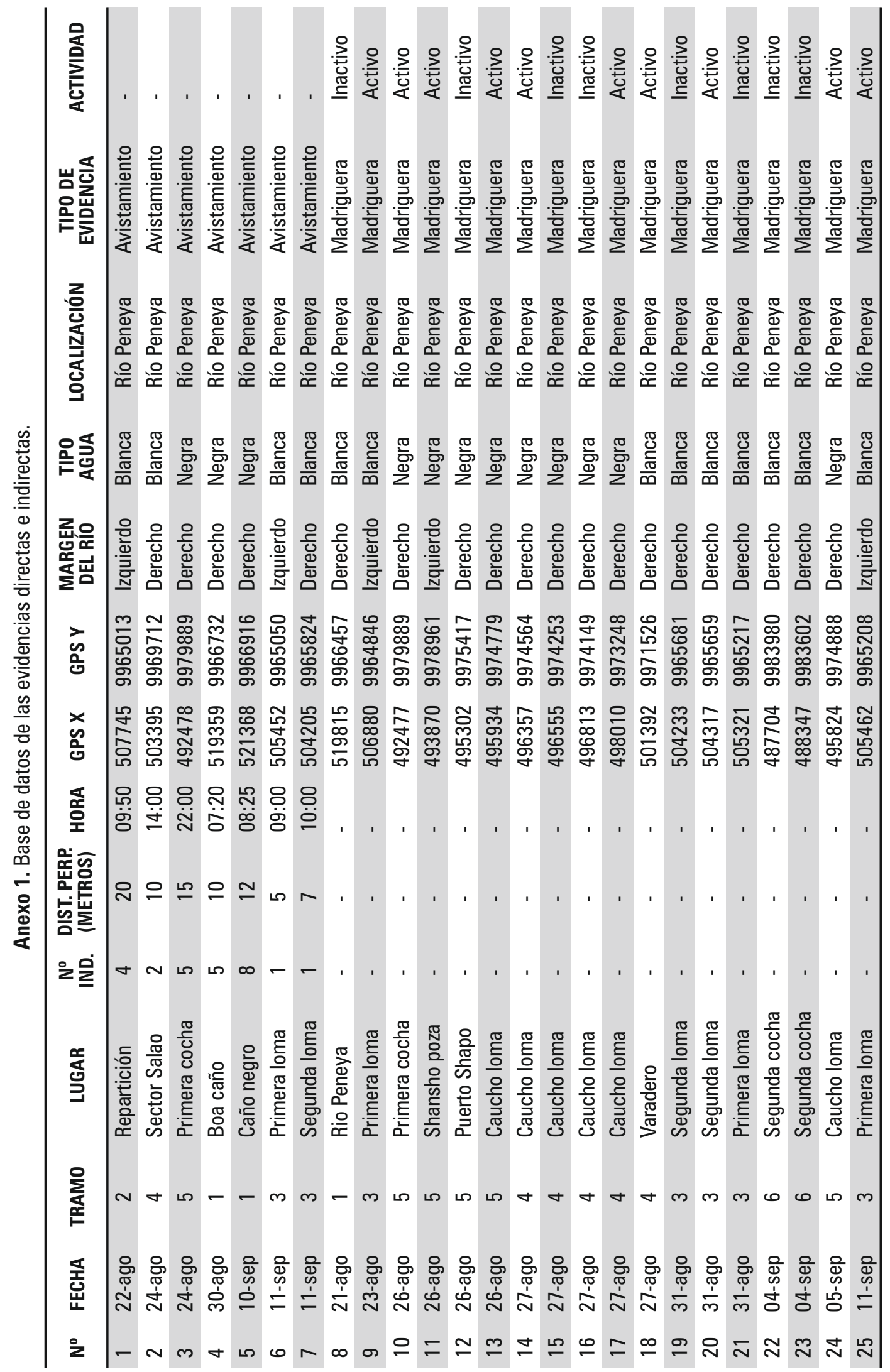




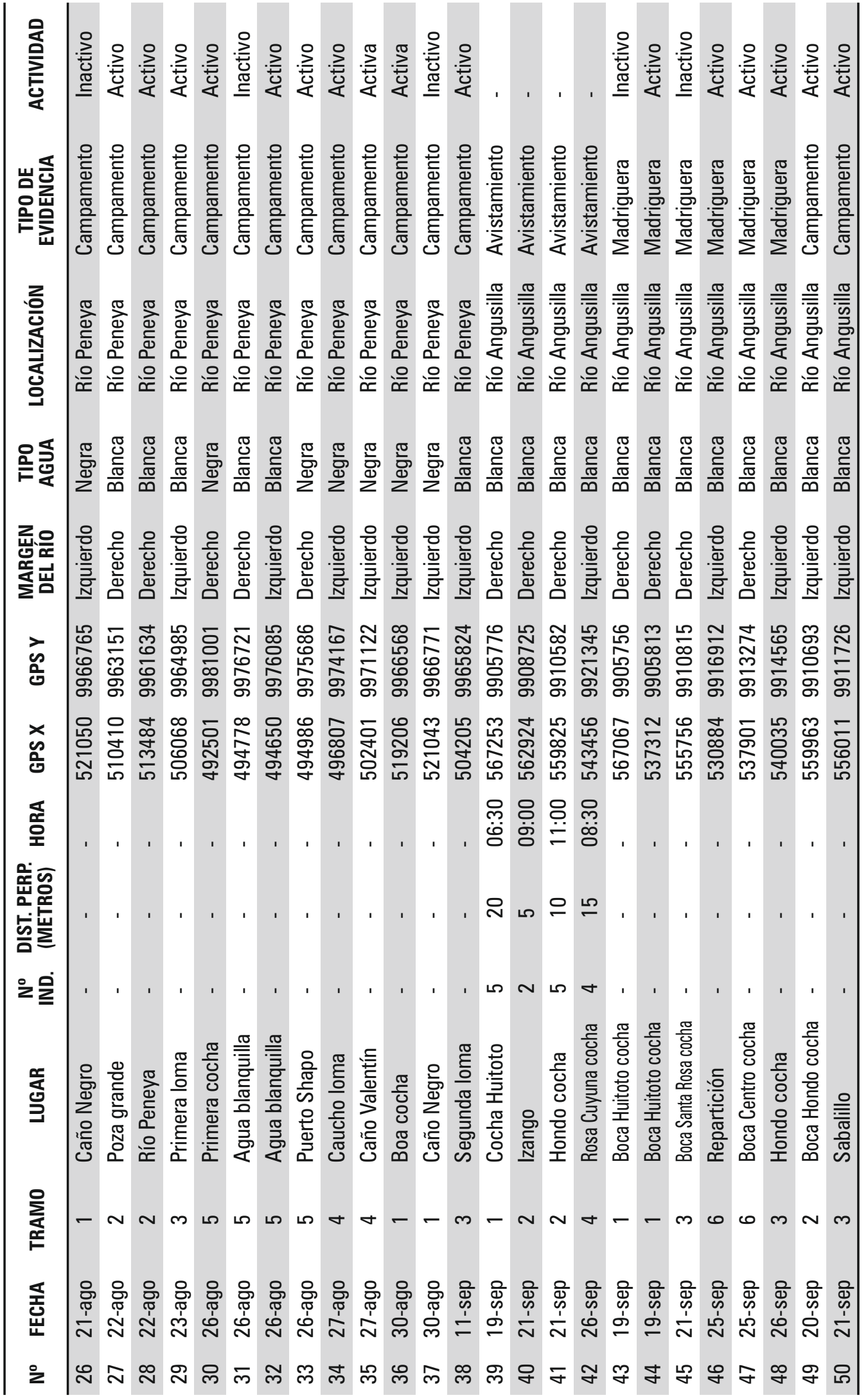


